\title{
Automated Extraction of Drainage Channels and Roads through Deep Learning
}

\author{
Lawrence Stanislawski $^{\text {a, }}{ }$, Tyler Brockmeyer ${ }^{\text {a }}$, Ethan Shavers ${ }^{\text {a }}$ \\ ${ }^{a}$ U.S. Geological Survey, Center of Excellence for Geospatial Information Science, Rolla Missouri, United States, lstan@usgs.gov, \\ TylerBrockmeyerr, tbrockmeyer@contractor.usgs.gov,Ethan Shavers,eshavers@usgs.gov \\ * Corresponding author
}

Keywords: hydrography, deep learning, drainage line extraction, road extraction

\begin{abstract}
:
The National Map provides geospatial data that support various uses such as resource management, disaster response, and science investigations. To properly support these needs, data themes of the National Map must be regularly updated and spatially integrated as the features on the ground change because of environmental or man-made events. The elevation theme of the National Map is managed through the 3D Elevation Program (3DEP), which is currently (2019) coordinating collection of high resolution (HR) elevation data for the United States over an eight-year period (Sugarbaker et al. 2014). Through this program, lidar point-cloud data are being collected for the conterminous United States, Hawaii, and U.S. territories, with coarser resolution interferometric synthetic aperture radar (ifsar) data being collected for the remote areas of Alaska. HR digital elevation models (DEMs) can be generated at 1 and 3 meter resolution from the lidar point-cloud data and are also furnished by 3DEP.
\end{abstract}

This research develops automated methods to update the hydrography and transportation themes of the National Map in a manner that integrates with the HR elevation and image layers. Surface water drainage networks can be extracted from a HR DEM using flow-direction and flow-accumulation modelling, but results of these methods vary depending on environmental conditions and the existence of anthropogenic features that may affect the accuracy of the elevation model, such as vegetative cover, roads, bridges, and other urban structures. Hydrologic conditioning or enforcement of a HR DEM overcome some of these issues and improve flow modelling for drainage network extraction through techniques such as filtering (Passalaqua et al. 2010), filling sinks (Tarboton 1997), cutting channels through embankments at culvert and bridge locations (Poppenga et al. 2012), or burning-in existing streams (Maidment 1996). However, drainage network extraction results can vary substantially with these techniques and the methods generally require some manual intervention and/or tuning of parameters (Poppenga et al. 2013). Consequently, additional work is needed to streamline and further automate such methods for the various landscape conditions within the United States.

Recent advances in machine learning techniques show promise for extracting water features from multispectral image data (Mishra and Prasad 2015, Yang et al. 2015, Yu et al. 2017). Chen et al. (2018) demonstrate high precision extraction of non-occluded waterbody features in urban areas. The authors use image segmentation and a convolutional neural network (CNN) on HR satellite data (approximately 4 to 6 meter resolution) from the visible and near-infrared wavebands. However, limited machine learning research has been devoted to extracting surface water drainage channels in places where little, if any, water may exist, such as in the headwater drainage channel features, or for features that may be occluded by tree canopy.

Here we present ongoing research to extract surface water features, focusing on drainage channels, using a CNN on data derived from lidar point-cloud and multiband image data. A primary dataset used in the CNN is the topographic position index (TPI). TPI is determined as the difference between a point elevation value and the local average elevation within a specific radius or within a surrounding window of cells (De Reu et al., 2013). The TPI exaggerates local lows and highs in a DEM relative to the nearby topographic features, and thus accentuates ridges and valleys. Roelens et al. (2018) demonstrate methods to extract connected drainage networks in an agricultural and residential area from 1-meter resolution DEM data derived from dense (16 points per square meter) lidar point-cloud data. In their work, initial drainage channels are extracted from a relative elevation dataset derived from the DEM, and then initial network segments are connected based on a connection probability model (Roelens et al. 2018). Relative elevation applied in that work is identical to TPI. Additional raster data layers that are being tested in the CNN include but are not limited to elevation, geometric curvature (Passalaqua et al. 2012), average lidar point intensity, and near-infrared reflectance from 1-meter resolution orthorectified color-infrared aerial photography furnished through National Agricultural Imagery Program.

Given that CNN methods have been successfully applied to extract road features (Sameen and Pradhan 2017), CNN models are tested to simultaneously predict drainage line and road pixels, and non-stream, non-road (NSNR) pixels. Training window patterns for roads and drainage lines are respectively derived from Census road and HR National 
Hydrography Dataset (NHD) flowline data that are available in the National Map. Road vectors for a study area are rasterized, and a set of road-pattern windows, each centered on a road pixel, is randomly sampled from the rasterized road dataset. A similar set of drainage-line-pattern windows is formed from drainage-line vector features. However, because NHD flowlines may not precisely follow drainage valleys in the HR DEM, a set of vector drainage lines that conform to DEM valleys is extracted from the HR DEM using Geonet flow-accumulation modelling software (Passalacqua et al. 2012). Drainage-line-pattern windows are formed from the DEM-extracted drainage lines that overlap with HR NHD flowlines and are therefore more likely to contain water than non-overlapping DEM-extracted lines. Each sample window is rotated multiple times to automatically form additional road and drainage-line patterns that may exist at different angles.

Center pixels for NSNR pattern windows are randomly sampled from pixels outside of buffers created around the vector roads and DEM-extracted drainage lines. Likewise, each sampled NRNS-pattern is rotated the same number of times as the road and drainage-line windows to form additional patterns that may exist at different angles. Rotating pattern windows is a fast and easy way to expand the set of sample patterns for recognition of similar patterns with different orientations. However, given that methods automatically select samples, it may be better to select more sample patterns of features that actually exist, rather than forming rotated patterns for features that may or may not exist.

All sample patterns for the three feature types (drains, roads, NSNR) are divided into three groups with equal numbers from each feature type. Two-thirds of the samples are used for CNN training. The remaining one-third is equally divided into two groups for validation and testing.

Methods are currently being tested for two agricultural areas in Iowa and Illinois to compare solutions from 1 and 3 meter resolution datasets. Aside from evaluating the different combinations of data layers and data layer resolution, several parameters (number of sample patterns, pattern window size, number of rotations of each pattern, buffer size around vector features for defining NSNR pixels) are being studied to identify an optimal solution. In addition, automated methods are being tested to subdivide the data into smaller data chunks for more efficient simultaneous processing of multiple CNN solutions, and these are being compared to a non-divided solution. The workflow is primarily implemented through Python programming and open source tools including Tensorflow ${ }^{\mathrm{TM}}$ and Keras.

Preliminary results for the Panther Creek agricultural watershed in central Iowa accurately predict better than 90 percent of the test samples using a CNN applied to only the TPI data layer (Stanislawski et al. 2018). The CNN in this case used 6000 41x41-pixel window patterns for the sample set (2000 of each type) with 3-meter resolution data. It is noted that an effective filtering and vectorization process could further improve the solution. Additionally, methods are needed to validate predicted features that are not included in the sample data. This task will likely involve some manual interpretation of HR image or other data in conjunction with flow accumulation modelling.

This research will develop and test fully automated workflows to update and, or validate hydrography and transportation features in the United States National Map using deep learning CNN modelling on existing National Map elevation and image data, and other readily available datasets. The workflow uses existing vector hydrography and road data to model and extract patterns for these features in the raster image and elevation datasets, or datasets derived therefrom. As relatively up-to-date lidar point-cloud and HR DEM data become available for all areas of the United States through 3DEP, pattern recognition and extraction workflows, as described here, may be an efficient alternative for maintaining current, accurate, and integrated data within the National Map. 